\title{
BMJ Open Enteric Salmonella in humans and food in the Middle East and North Africa: protocol of a systematic review
}

\author{
Karima Chaabna, ${ }^{1,2}$ Walid Alali ${ }^{3}$
}

To cite: Chaabna K, Alali W. Enteric Salmonella in humans and food in the Middle East and North Africa: protocol of a systematic review. BMJ Open 2017;7:e017399. doi:10.1136/ bmjopen-2017-017399

- Prepublication history and additional material for this paper are available online. To view these files please visit the journal online (http://dx.doi. org/10.1136/bmjopen-2017017399).

Received 20 April 2017 Revised 6 June 2017 Accepted 6 June 2017

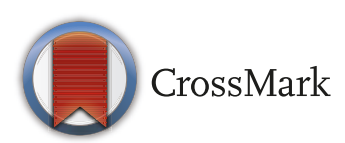

${ }^{1}$ Infectious Disease Epidemiology Group, Weill Cornell Medical College in Qatar, Cornell University, Doha, Qatar

${ }^{2}$ Department of Healthcare Policy and Research, Weill Cornell Medical College, Cornell University, New York, USA

${ }^{3}$ College of Public Health, Hamad bin Khalifa University, Doha, Qatar

Correspondence to Dr Karima Chaabna; chaabnak.epi@gmail.com

\begin{abstract}
Introduction Non-typhoidal Salmonella is considered one of the leading causes of foodborne disease worldwide. This protocol provides methods that will be used to synthesise available epidemiological data on non-typhoidal enteric Salmonella in humans and food in Middle East and North Africa (MENA) region and to characterise the morbidity of human salmonellosis in this region.

Methods and analysis A systematic review will be conducted based on the Cochrane Collaboration handbook and will be reported following the items outlined in the Preferred Reporting Items for Systematic Reviews and Meta-Analyses guidelines. We will search PubMed, Embase, CAB Direct and Global Health Library (WHO) databases in order to identify relevant reports. Additionally, the literature search will be supplemented by checking references of the included reports and the identified reviews. Furthermore, we will hand-search conference proceedings and Ministry of Health's website of each country of the MENA region. We will use comprehensive search criteria with no time and no language restrictions. We will extract data on report and study characteristics, biological assay characteristics, individuals' demographic characteristics and on primary and secondary outcomes of interest. If appropriate, meta-analysis will be conducted in order to estimate pooled prevalence measures using DerSimonian and Laird random-effects models. We will conduct meta-regression analysis to explore the effect of study-level characteristics as potential sources of heterogeneity.
\end{abstract}

Ethics and dissemination The results of the systematic review will be disseminated in a peer-reviewed journal and presented at relevant conferences.

Trial registration number The trial registration number is CRD42016046360.

\section{INTRODUCTION}

Non-typhoidal Salmonella is considered one of the leading causes of foodborne disease worldwide. WHO estimated that the annual median number of non-typhoidal salmonellosis was 78.7 million foodborne illnesses with over 59000 deaths. ${ }^{1}$ As for the WHO-defined Eastern Mediterranean Region, the median incidence rate of non-typhoidal salmonellosis was 1610 illnesses with 0.6 death and disability-adjusted life years (DALYs) was 54

\section{Strengths and limitations of this study}

To the best of our knowledge, this is the first systematic review evaluating the epidemiology of non-typhoidal enteric Salmonella in humans and food in the countries of the Middle East and North Africa region.

- This systematic review will potentially inform policy makers in order to strengthen national foodborne disease surveillance and to improve food safety in this region.

- One of our limitations will be probably a high heterogeneity between studies related to sample size, populations, settings, study periods and the use of different biological assays to ascertain the infection.

per 100000 persons, whereas the median incidence rate in the WHO-defined African Region is 896 illnesses with 1 death and 89 DALYs per 100000 persons. $^{2}$

Countries in the Middle East and North Africa (MENA) region share similar heritage, religion and language. However, the socioeconomic status, governance, growth and development and healthcare system in the MENA region differ widely. Although foodborne disease outbreaks have been frequently reported in the MENA region, a rigorous reporting and monitoring system (ie, active surveillance system) is lacking to quantify the incidence/prevalence of foodborne pathogens and disease. Nonetheless, published studies from the MENA region have reported data on foodborne disease morbidity in human populations. Furthermore, data on the prevalence of food contaminants have been revealed in MENA countries. Non-typhoidal Salmonella species are a common cause of foodborne disease in the MENA region. ${ }^{1}$ Moreover, Salmonella has been detected in an array of food products presented to consumers in the region. The number and quality of the studies differ substantially by country. To the best of our knowledge, there has been no published study 
that systematically reviewed, synthetised and assessed the available data on non-typhoidal enteric Salmonella in humans and food in the MENA region. Synthetising the data in addition to characterising the morbidity of human salmonellosis in MENA will provide a rational basis for source attribution studies at regional and country level. Additionally, this study will inform policy maker in order to strengthen national foodborne disease surveillance, improve food safety and prioritise food control intervention programs.

\section{OBJECTIVES}

The proposed systematic review will identify, synthetise and assess the available data on non-typhoidal enteric Salmonella in humans and food in each country of the MENA region. Therefore, our review will address the following questions: (1) What is the non-typhoidal salmonellosis morbidity in human populations in MENA? (2) What is the non-typhoidal Salmonella prevalence in food in MENA? (3) What is the distribution of Salmonella serotypes in human populations and food?

\section{METHODS AND ANALYSIS}

This systematic review protocol was developed based on the Cochrane Collaboration handbook ${ }^{3}$ and reported following the statement outlined by Preferred Reporting Items for Systematic Review and Meta-Analysis Protocols (PRISMA-P) 2015 statements. ${ }^{4}$ PRIMSA-P 2015 checklist ${ }^{5}$ was completed and can be found in the research checklist.

\section{Inclusion and exclusion criteria \\ Types of studies}

All reports meeting the inclusion criteria will be included if the study sample size is higher than 10. Case reports, case series, expert opinion, reviews, original articles reporting qualitative and experimental studies, editorials, commentaries, letters to editors, author replies and newspaper articles will be excluded.

\section{Type of participants}

Included reports are those studying humans and food. Reports will be excluded if the studies were on enteric Salmonella in live food-producing or domestic animals as well as in water, fomite, soil or other environments.

\section{Types of exposures}

Included reports are those studying non-typhoidal enteric Salmonella. These reports need to present studies that used laboratory testing for Salmonella ascertainment. More precisely, in humans, the laboratory testing has to be conducted on stool samples; that is, reports of studies based on clinical diagnosis without any laboratory tests on stool to confirm the causative agent will be excluded. Therefore, reports on non-typhoidal enteric Salmonella from gastro-intestinal tract infections will be included, while reports on respiratory, urinary tract and bloodstream infections will be excluded. Additionally, studies on non-typhoidal enteric Salmonella cultured from cerebrospinal fluid will be excluded. Reports referring to non-typhoidal enteric Salmonella infection as Salmonella infection or as salmonellosis will be included, whereas those referring to enteric Salmonella as typhoidal, paratyphoidal or invasive non-typhoidal Salmonella infection (that is not foodborne or cause of gastro-intestinal tract infections) will be excluded.

\section{Types of outcomes}

Our primary outcomes are non-typhoidal enteric Salmonella morbidity (prevalence), serotype distribution, bacteria attributable mortality and all-cause mortality in human populations, hospitalisation and length of stay in hospital. Our secondary outcomes are enteric Salmonella prevalence and serotype distribution in food.

\section{Data sources and search strategy}

Our systematic review will be conducted based on the Cochrane Collaboration handbook ${ }^{3}$ and will be reported following the items outlined in the Preferred Reporting Items for Systematic Reviews and Meta-Analyses (PRISMA) statement. We will search PubMed, ${ }^{6}$ Embase, ${ }^{7}$ CAB Direct $^{8}$ and Global Health Library $(\text { WHO })^{9}$ databases in order to identify further relevant reports (online supplementary file 1). In addition, the literature search will be supplemented by checking references of the included reports and the identified reviews. Furthermore, we will hand-search conference proceedings and Ministry of Health's website of each country of the MENA region. We will use comprehensive search criteria with no time and no language restrictions. We will construct our search criteria using Boolean logic (OR and AND) to combine Medical Subject Headings (MeSH) terms and text words. Key search terms will include countries' names, MENA populations' names and Salmonella. We will use WHO/ Eastern Mediterranean Region (EMR ${ }^{10}$ definition of MENA region and we will complement this list with four countries whose official languages are Arabic ${ }^{11}$ and that are cited in other definitions of MENA. ${ }^{12-14}$ The reviewer team does not speak the official language of Cyprus ${ }^{11}$ or the media of instruction in its universities and colleges ${ }^{15}$; this will prevent us to identify grey literature such as reports from the Ministry of Health, journal articles and conference abstracts published in these languages. As such, we decide to exclude this country. Our systematic review will include 24 countries, namely: Afghanistan, Algeria, Bahrain, Djibouti, Egypt, Iran, Iraq, Jordan, Kuwait, Lebanon, Libya, Morocco, Mauritania, Oman, Pakistan, Palestine, Qatar, Saudi Arabia, Somalia, Sudan, Syria, Tunisia, United Arab Emirates and Yemen. The selected MENA countries have a total population of more than 680 million people. ${ }^{16}$

\section{Study records}

\section{Selection process}

Identified references will be imported into a reference manager $\left(\right.$ Endnote $^{17}$ ) where duplicate reports will be excluded. The title and abstract screening for relevance, followed by the full-text screening of the unique reports, 
will be conducted by KC. This multi-level screening process will be checked by WA. Any disagreements will be resolved by discussion and consensus. Non-eligible reports will be excluded, and the reasons for their exclusion will be recorded.

\section{Data collection process}

A piloted standardised form developed in Microsoft Excel $2010^{18}$ by $\mathrm{KC}$ and WA will be used for the extraction step. Extraction of relevant data will be done by $\mathrm{KC}$, and $25 \%$ of the data will be checked for correctness by WA.

\section{Data items}

We will extract data on report characteristics (authors, year of publication, title, etc), study characteristics (year of data collection, study site and design, sampling methodology, prevalence, number of positive cases, sample size, etc), biological assay characteristics, individuals' demographic characteristics (age, gender, etc) and on primary and secondary outcomes of interest.

\section{Risk of bias in individual studies}

Based on the Cochrane approach, ${ }^{3}$ the risk of bias (ROB) assessment will be conducted at both the study level and the outcome level. Each study will be classified as having a low, high or unclear ROB in each of the three quality domains, namely, sampling methodology, infection ascertainment and response rate. An ROB will be considered low if these three quality domains are probability based, ascertainment by biological assays or response rate is $\geq 80 \%$, respectively. At outcome level, a minimum sample size will be calculated using exact binomial CI formula ${ }^{19}$ in order to differentiate outcome measures with good precision. Sample size of studies considered as having good precision should be equal or higher than the minimum sample size defined in this protocol.

\section{Data synthesis}

We will report our systematic review following Preferred Reporting Items for PRISMA 2009 statements ${ }^{20}$ and PRISMA for Abstracts Checklist. ${ }^{21}$ We will qualitatively synthesise the identified data on non-typhoidal enteric Salmonella in humans and food. These data will be stratified by country and according to the clinical status of the study populations:

1. Non-clinical populations in community settings: healthy populations, mainly food workers

2. Clinical populations: patients with diarrhoea due to gastro-intestinal pathogenic microbes

In addition, a third stratum will be created for the food category. According to the diversity of the identified population subgroups, we will decide if we also need to create subcategories in each stratum.

If data are appropriate for quantitative synthesis, data analyses will be conducted in R V.3.1.1 $1^{22}$ using the meta ${ }^{23}$ and metafor ${ }^{24}$ packages. Using meta-analysis, we aim to estimate pooled prevalence of Salmonella in food (stratified by category: poultry, beef and seafood, etc) and in human (stratified by type of population). Outcome measures will be pooled in all strata with at least three outcome measures included. Meta-regression will be used in order to assess heterogeneity across studies ${ }^{3}$ related to sample size, populations, settings, study periods and the use of different biological assays to ascertain the infection. Additionally, we will conduct sensitivity analysis restricted to studies at low ROB in order to explore the impact of high ROB study measures on the pooled estimates.

\section{Meta-bias}

Regarding meta-bias assessment, we will use funnel plots in order to explore small-study effect on the pooled estimates. ${ }^{25}$ Traditional funnel plots (log (odds proportion) vs $1 / \mathrm{SE}$ ) are inaccurate for meta-analysis of proportion studies. Therefore, we will create funnel plots of $\log$ (odds proportion) against sample size. ${ }^{26}$ In order to test the asymmetry of the funnel, we will perform Egger test, ${ }^{25}$ which is based on SE, as well as Peter test, which is based on sample size. ${ }^{26} 27$

\section{Confidence in cumulative evidence}

We will use a narrative justification for the quality of the evidence at the country level. We will consider the quality of evidence being better in a country if at least one country-level study was conducted. This country-level study should have used standard methodology including probability-based sampling. Thus, we will categorise countries as having:

- Poor evidence: poor quality of the outcome measures

- Limited evidence: the number of outcome measures is small but of reasonable quality

- Good evidence: the number of outcome measures is small but with good quality

- Conclusive evidence: enough outcome measures with good quality

\section{DISCUSSION}

To the best of our knowledge, this systematic review will be the first attempt to synthetise available data on non-typhoidal enteric Salmonella in humans and food in the countries of the MENA region and to characterise the morbidity of human salmonellosis. This work will enable us to identify key pathogen control points that should be reinforced and those that need to be further assessed through country-level studies. Ultimately, this systematic review will provide rational basis for source attribution studies at both regional and country levels. ${ }^{28}$ Additionally, this study will inform policy maker actions in order to strengthen national foodborne disease surveillance and to improve food safety and public health in MENA.

Contributors $\mathrm{KC}$ and WA contributed to the conception of the study. The manuscript protocol was drafted by KC and revised by WA. The search strategy was developed and will be conducted by both authors who will also screen the potential reports, extract data, assess the risk of bias and perform the data synthesis. Both authors approved the publication of the current protocol.

Funding This research received no specific grant from any funding agency in the public, commercial or not-for-profit sectors.

Competing interests None declared.

Provenance and peer review Not commissioned; externally peer reviewed. 
Open Access This is an Open Access article distributed in accordance with the Creative Commons Attribution Non Commercial (CC BY-NC 4.0) license, which permits others to distribute, remix, adapt, build upon this work non-commercially, and license their derivative works on different terms, provided the original work is properly cited and the use is non-commercial. See: http://creativecommons.org/ licenses/by-nc/4.0/

(c) Article author(s) (or their employer(s) unless otherwise stated in the text of the article) 2017. All rights reserved. No commercial use is permitted unless otherwise expressly granted.

\section{REFERENCE}

1. Havelaar AH, Kirk MD, Torgerson PR, et al. World Health Organization Global estimates and regional comparisons of the burden of foodborne disease in 2010. PLoS Med 2015;12:e1001923.

2. Kirk MD, Pires SM, Black RE, et al. World Health Organization estimates of the global and regional disease burden of 22 foodborne bacterial, protozoal, and viral diseases, 2010: a data synthesis. PLoS Med 2015;12:e1001921.

3. Higgins JPT, Green S. Cochrane Handbook for Systematic Reviews of Interventions, 2008.

4. Moher D, Shamseer L, Clarke M, et al. Preferred reporting items for systematic review and meta-analysis protocols (PRISMA-P) 2015 statement. Syst Rev 2015;4:1.

5. Shamseer L, Moher D, Clarke M, et al. Preferred reporting items for systematic review and meta-analysis protocols (PRISMA-P) 2015: elaboration and explanation. BMJ 2015;349:g7647.

6. National institutes of Health. PubMed.gov US National Library of Medcine. Bethesda: USAN.D. http://www.ncbi.nlm.nih.gov/pubmed.

7. Ovid Technologies i. USA: Emabase New York, 2016. http://ovidsp.tx. ovid.com/sp-3.21.1b/ovidweb.cgi?\&S=GLEEFPFGFJDDNDAFNCIKM HGCJCKEAA00\&New+Database=Single\%7c6. (accessed $15 \mathrm{Feb}$ 2017).

8. CAB International. CAB Direct United Kingdom,2016; https://www. cabdirect.org/ accessed 25 Dec 2016.

9. World Health Organization Global Health Library Geneva, Switzerland N.D. [: http://www.globalhealthlibrary.net/php/index.php accessed 1 Jan 2017.

10. World Health Organization. Country groupings: subregional country groupings for the global assessment of disease burden: WHO. N.D. http://www.who.int/quantifying_ehimpacts/global/ebdcountgroup/ en/. (accessed 25 Jan 2017).
11. The Central Intelligence Agency. The World Factbook: The Central Intelligence Agency, 2017. https://www.cia.gov/library/publications/ the-world-factbook/. (accessed 26 Jan 2017).

12. The World Bank. The World Bank: Data: Wold Bank Country and Lending Groups, 2017. https://datahelpdesk.worldbank.org/ knowledgebase/articles/906519-world-bank-country-and-lendinggroups. (accessed Jul 25 2017).

13. UNAIDS. UNAIDS Middle East \& North Africa: UNAIDS, 2017. http:// www.unaidsmena.org/. (accessed 25 Jan 2017).

14. Institute for Health Metrics and evaluation GBD compare |Viz Hub. University of Washington, 2017. http://vizhub.healthdata.org/gbdcompare/. (accessed 15 Feb 2017).

15. L'Europe Cde. Language Education Policy Profile: Cyprus: Conseil de 1 L'Europe, N.D.

16. Nations U. Total Population - Both sexes. De facto population in a country, area or region as of 1 July of the year indicated. Figures are presented in thousands. World Population Prospects: The 2015 Revision 2016 https://esa.un.org/unpd/wpp/Download/Standard/ Population/ (accessed 1 Jan 2017).

17. Reuter Thomson. Endnote X7 2016

18. Microsoft. Microsoft Excel 2010, 2010.

19. Clopper CJ, Pearson ES. The use of confidence or fiducial limits illustrated in the case of the binomial. Biometrika 1934;26:404-13.

20. Moher D, Liberati A, Tetzlaff J, et al. Preferred reporting items for systematic reviews and meta-analyses: the PRISMA statement. $J$ Clin Epidemiol 2009;62:1006-12.

21. Beller EM, Glasziou PP, Altman DG, et al. PRISMA for abstracts: reporting systematic reviews in journal and conference abstracts. PLoS Med 2013;10:e1001419.

22. The R Project for Statistical Computing. R-3.3.1 for Windows (32/64 bit): The R Foundation, 2016. https://cran.r-project.org/bin/windows/ base/. (accessed Aug 30).

23. Schwarzer G. Meta package 2016. https://cran.r-project.org/web/ packages/meta/meta.pdf. (accessed Aug 30 2016).

24. Schwarzer G. Metafor package 2015. https://cran.r-project.org/web/ packages/metafor/metafor.pdf. (accessed Aug 30 2016).

25. Sterne JA, Egger M. Funnel plots for detecting bias in meta-analysis: guidelines on choice of Axis. J Clin Epidemiol 2001;54:1046-55.

26. Hunter JP, Saratzis A, Sutton AJ, et al. In meta-analyses of proportion studies, funnel plots were found to be an inaccurate method of assessing publication Bias. J Clin Epidemiol 2014;67:897-903.

27. Moreno SG, Sutton AJ, Ades AE, et al. Assessment of regressionbased methods to adjust for publication bias through a comprehensive simulation study. BMC Med Res Methodol 2009;9:2.

28. Ahlstrom C, Muellner P, Spencer SE, et al. Inferring source attribution from a multiyear multisource data set of Salmonella in Minnesota. Zoonoses Public Health 2017. 